\title{
Untersuchungen über die chemische Zusammensetzung und Bildung der Enzyme.
}

VIII. Mitteilung.

Über die gleìchzeitige Veränderung des Gehaltes an Invertase und an Gärungsenzymen in der lebenden Hefe.

Von

Hans Euler und David Johansson.

Mit drei Kurvenzeichnungen im Text.

(Aus dem biochemischen Laboratorium der Hochschule Stockholm.) .

(Der Redaktion zugegangen am 10. Februar 1913.)

Die Veränderung der enzymatischen Rohrzuckerspaltung in lebender Hefe wurde in den vorhergehenden Untersuchungen von uns ${ }^{1}$ ) sowie von Euler und Meyer ${ }^{2}$ ) quantitativ studiert, sowohl hinsichtlich des zeitlichen Verlaufs der Zunahme der enzymatischen Wirksamkeit einer gewissen Hefenmenge unter dem Einfluß einer Vorbehandlung, als auch hinsichtlich des Einflusses derjenigen Stoffe, mit welchen die Hefe vorbehandelt wird.

Gegenüber der «Enzymbildung》 bei der Galaktosevergärung ${ }^{3}$ ) ergibt sich der Unterschied, daß in obigem Falle das Substrat nicht dem gebildeten Enzym zu entsprechen braucht. Es wurde demgemäß sicher kein größerer Zuwachs an rohrzuckerspaltender Wirkung beobachtet, wenn eine Vorbehandlung der Hefe mit Rohrzucker stattfand, als wenn die Hefe mit Glukose vorbehandelt wurde. Eine Gewöhnung an das Substrat bezw. eine Anpassungserscheinung lag -also bei der von uns beobachteten enzymatischen Veränderung der invertierenden Wirkung nicht vor.

1) Euler und Johansson, Diese Zeitschrift, Bd. 76, S. 388, 1912.

2) Euler und Meyer, Diese Zeitschrift, Bd. 79, S. 274, 1912.

3) Euler und Johansson, Diese Zeitschrift, Bd. 78, S. 246, 1912. 
I.

In Rücksicht auf die Versuche, welche L. Lichtwitz ${ }^{1}$ ) nach dem Erscheinen der Arbeiten von B. af Ugglas, Kullberg und uns veröffentlicht hat, wurden noch weitere Untersuchungen darüber angestellt, ob der Zuwachs der invertierenden Wirkung, welche lebende Hefe beim Verweilen in gewissen zuckerhaltigen Nährlösungen erfährt, von der Gegenwart der Spaltprodukte des Rohrzuckers (Glukose und Fruktose) in der Kulturflüssigkeit abhängig ist.

Unsere diesbezüglichen ergänzenden Versuche bezogen sich auf einen Vergleich des Einflusses der Fruktose mit dem des Rohrzuckers.

Es ist zunächst hervorzuheben, daß die Art der Vorbehandlung bei den Versuchen von Lichtwitz eine andere ist als bei unseren Versuchen. ${ }^{2}$ ) Lichtwitz impft nämlich die Hefe in die verschiedenen Zuckerlösungen ein und läßt die Hefe sich darin entwickeln.

Die Hefe (Reinkultur der Weinhefe, Rasse ¿Oppenheimer Kreuz» wurde von Lichtwitz in zwei Reihen gezüchtet. In der Reihe a enthielt die Nährlösung (Hefewasser) 10\% Rohrzucker, in der Reihe b $10 \%$ lnvertzucker. Mit diesen beiden Hefen wurde, nachdem sie so längere Zeit gezogen waren, große Kulturen angelegt, welche bestimmte Mengen Rohrzucker,..Invertzucker, oder Mischungen beider enthielten. Mit den auf Rohrzucker und den auf Invertzucker gewachsenen Zellen wurden dann vergleichende Gärungs- und Inversionsversuche angestellt.

Die in Invertzucker gewachsenen Zellen zeigten bei den Versuchen von Lichtwitz tatsächlich eine geringere invertierende Fähigkeit als diejenigen, welche sich in Gegenwart von Rohrzucker entwickelt hatten.

Im Gegensatz hierzu ergaben auch unsere neuen Versuche das Ergebnis, da $B-$ bei unserer, auch hier beibehaltenen Versuchsanstellung - die Spaltpro-

1) Diese Zeitschrift, Bd. 78, S. 129, 1912.

$\left.{ }^{2}\right)$ Dagegen war die von Lichtwitz angewandte Versuchsmethodik zur Ermittlung der invertierenden Wirkung der lebenden Zellen ganz analog der von Euler und Kullberg (Diese Zeitschrift, Bd. 71, S. 20, 1911) angegeben; vgl. daselbst den Abschnitt: Zur Dynamik der Enzymreaktionen mittels Hefezellen. 
dukte des Rohrzuckers keinen spezifischen Einfluß auf das Inversionsvermögen der Hefezellen ausüben.

$\mathrm{Daß}$ es sich bei den Versuchen von Lichtwitz um eine „Fermentlähmung *, also eine Erscheinung handelt, analog der von Tammann ${ }^{1}$ ) in seiner grundlegenden Arbeit beschriebenen, ist allerdings äußerst unwahrscheinlich, wie wir denn überhaupt auf die theoretische Behandlung der Erörterungen, welche Lichtwitz seinen an sich interessanten Versuchen angefügt hat, noch zurückkommen müssen.

II.

In der erwähnten Arbeit von Euler und Meyer wurde zwischen spezifischer und genereller Enzymbildung unterschieden.

Eine spezifische Enzymbildung tritt bei der Anpassung von Zellen an gewisse Nahrungsstoffe ein, eine generelle Enzymbildung überall da, wo es sich um eine allgemeine Kräftigung der Zellen handelt.

Es wurde ferner die Vermutung ausgesprochen, daß bei der im hiesigen Laboratorium studierten Erhöhung des Inversionsvermögens durch Vorbehandlung vielleicht eine generelle Enzymbildung vorliegt, wobei bereits betont wurde, daß allerdings von einer Parallelität der Zunahme nicht die Rede sein kann.

Um über die Art der «Invertasebildung» Aufschluß zu gewinnen, wurde in der vorliegenden Intersuchung die unter gewissen Bedingungen vorbehandelte Hefe gleichzeitig auf ihre Invertasewirkung und ihre Gärungswirkung untersucht.

\section{Versuchsanordnung.}

Zur Vorbehandlung wurden $5 \mathrm{~g}$ der abgepreßten gewaschenen Hefe in $500 \mathrm{ccm}$ sterilisierter Haydukscher Nährlösung eingetragen; welche im Liter enthielt:

$$
\begin{aligned}
& 0,25 \mathrm{~g} \mathrm{MgSO}_{4} \quad 5 \mathrm{~g} \mathrm{KH}_{2} \mathrm{PO}_{4} \\
& 4 .\left(\mathrm{NH}_{4}\right)_{2} \mathrm{SO}_{4} \quad 20 \text {, Rohrzucker. }
\end{aligned}
$$

2) Diese Zeitschrift, Bd. 16, S. 271, 1892. - Zeitschrift f. physik. Chem., Bd. 18, S. 426, 1895. 
Diese Zusammensetzung der Nährlösung gilt für alle Versuche, welche in den Figuren 1 und 2 zusammengefaßt sind, und außerdem für die Versuche der Kurve 3 in Fig. 3.

Die Vorbehandlung der Hefe in den Versuchen der Kurve 1 Fig. 3 geschah in einer Nährlösung, welche weniger Stickstoff enthielt als die früher angewandte. Die Zusammensetzung war per Liter:

$$
\begin{aligned}
& 0,25 \mathrm{~g} \mathrm{MgSO}_{4} \quad \text { 5 } \mathrm{g} \mathrm{KH}_{2} \mathrm{PO}_{4} \\
& 1 \text {. } \mathrm{NH}_{4} \mathrm{H}_{2} \mathrm{PO}_{4} 20 \text {. Rohrzucker. }
\end{aligned}
$$

Die in der Kurve 2, Fig. 3 dargestellte Hefe wurde mit folgender Lösung vorbehandelt:

$$
\begin{aligned}
& 0,25 \mathrm{~g} \mathrm{MgSO}_{4} \quad 5 \mathrm{~g} \mathrm{KH}_{2} \mathrm{PO} \\
& 0,25 \text { * } \mathrm{NH}_{4} \mathrm{H}_{2} \mathrm{PO}_{4} \quad 20 \text { * Rohrzucker. }
\end{aligned}
$$

Ein Parallelversuch mit einer Nährlösung von der $\mathrm{Zu}$ sammensetzung:

$$
\begin{array}{ll}
0,25 \mathrm{~g} \mathrm{MgCl} & 5 \mathrm{~g} \mathrm{KH}_{2} \mathrm{PO}_{4} \\
0,25 & \text { » } \mathrm{NH}_{4} \mathrm{H}_{2} \mathrm{PO}_{4} \quad 20 \text { » Rohrzucker }
\end{array}
$$

ergab gegenüber dem vorhergehenden Versuch keinen Unterschied. Wie bei der Untersuchung von Euler und H. Meyer (diese Zeitschrift, Bd. 79, S. 274; 1912) wurden nun, mit einer und derselben Hefe als Ausgangsmaterial, teils Reihenversuche angestellt, bei welchen sich die Hefe während verschiedener Zeiträume in der Nährlösung befand (in der Fig. 1 sind die betreffenden Beobachtungen mit I, II, III, IV und V bezeichnet) teils wurden Abzweigungen gemacht, indem einem Kolben dieser ersten Serie Hefe entnommen und in eine neue, gleich große Menge Nährlösung verpflanzt wurde (Serie $\mathrm{II}_{1}, \mathrm{IV}_{1}$ und $\mathrm{V}_{1}$ ). Auch von dieser zweiten Reihe wurde wieder eine abgewogene Hefemenge weiter verpflanzt (Serie $\mathrm{II}_{1} \mathrm{a}$ ).

Die eine gewisse Anzahl Stunden vorbehandelte Hefe wurde abfiltriert und einige Minuten auf Ton getrocknet. Der dabei erreichte Gehalt an Trockensubstanz wurde jedesmal durch Entwässern eines Teiles der Hefe bei $95^{\circ}$ bestimmt. Von dieser Hefe wurden $0,25 \mathrm{~g}$ in $10 \mathrm{ccm} 1 \%$ iger $\mathrm{NaH}_{2} \mathrm{PO}_{4}$ Lösung aufgeschwemmt und nach 10 Minuten mit $20 \mathrm{ccm}$ einer $20 \%$ igen Rohrzuckerlösung versetzt. 
Zur Messung der. Inversionskraft der Hefe wurde nach bestimmten Versuchszeiten die gesamte Reaktion in jedem Kolben mit $10 \mathrm{ccm}$ einer $5 \%$ igen Sodalösung abgebrochen. Die Lösung wurde hierauf abfiltriert und im $1 \mathrm{dm}-R o h r$ polarisiert.

Bei den Gärversuchen wurde die Kohlensäure volumetrisch bestimmt. Die Übersättigung von Kohlensäure wurde vor jeder Ablesung durch anhaltendes energisches Schütteln aufgehoben. Bei allen Gärversuchen befand sich $0,5 \mathrm{~g}$ Hefe (von ca. 35\% Trockengewicht) in $25 \mathrm{ccm} 5 \%$ iger Rohrzuckerlösung. Versuchstemperatur bei der Gärung durchgehend $30^{\circ}$.

In allen Gärungstabellen und in allen Inversions- und Gärungskurven ist die Reaktionsgeschwindigkeit auf einen Trockengehalt von $35 \%$ reduziert. Die Inversionsversuche sind außerdem auf eine gemeinsame Versuchstemperatur $19^{\circ}$ reduziert.

(Tab. 1.)

0.

Ohne Vorbehandlung. Temperatur $20^{\circ}$.

\begin{tabular}{c|c|c}
\hline \hline Minuten & $\alpha$ & $\mathrm{k} \cdot 10^{4}$ \\
\hline 0 & 6,75 & - \\
15 & 5,29 & 52 \\
25 & 4,30 & 56 \\
35 & 3,62 & 52 \\
$\infty$ & $-2,16$ & -
\end{tabular}

(Tab. 2.)

I.

6 Stunden vorbehandelt.

Temp. $20^{\circ}$.

\begin{tabular}{c|r|r}
\hline \hline Minuten & \multicolumn{1}{|c|}{$\alpha$} & $\mathrm{k} \cdot 10^{4}$ \\
\hline 0 & 6,75 & - \\
15 & 4,35 & 91 \\
25 & 3,08 & 92 \\
35 & 1,88 & 98 \\
$\infty$ & $-2,16$ & -
\end{tabular}

Trockensubstanz: $32,9 \%$.
(Tab. 3.)

II.

24 Stunden vorbehandelt. Temp. $19,5^{\circ}$.

\begin{tabular}{c|r|r}
\hline Minuten & \multicolumn{1}{|c|}{$\alpha$} & $\mathrm{k} \cdot 10^{4}$ \\
\hline 0 & 6,75 & - \\
15 & 4,02 & 106 \\
25 & 2,54 & 111 \\
35 & 1,40 & 114 \\
$\infty$ & $-2,16$ & -
\end{tabular}

Trockensubstanz: $34,6 \%$.

Hoppe-Seylez's Zeitschrift f. physiol. Chemie. LXXXIV. 
(Tab. 4.) III.

16 Stunden vorbehandelt. Temp. $18,6^{\circ}$.

\begin{tabular}{c|r|c}
\hline Minuten & \multicolumn{1}{|c|}{$\alpha$} & $\mathrm{k} \cdot 10^{4}$ \\
\hline 0 & 6,75 & - \\
15 & 4,00 & 107 \\
25 & 2,55 & 111 \\
35 & 1,35 & 116 \\
$\infty$ & $-2,16$ & -
\end{tabular}

Trockensubstanz: $28,1 \%$.

(Tab. 6.)

V.

118 Stunden vorbehandelt. Temp. $1^{\circ}$.

\begin{tabular}{c|r|c}
\hline Minuten & \multicolumn{1}{|c|}{$\alpha$} & $\mathrm{k} \cdot 10^{4}$ \\
\hline 0 & 6,82 & - \\
15 & 3,32 & 143 \\
25 & 1,15 & 153 \\
35 & 0,38 & 156 \\
$\infty$ & $-2,18$ & - \\
\multicolumn{2}{|c}{ Trockensubstanz: $35,2 \%}$.
\end{tabular}

(Tab. 8.) $\quad \mathrm{V}_{1}$. $125+15$ Stunden vorbehandelt. Temp. $19^{\circ}$.

\begin{tabular}{c|r|c}
\hline \hline Minuten & \multicolumn{1}{|c|}{$\alpha$} & $\mathrm{k} \cdot 10^{4}$ \\
\hline 0 & 6,82 & - \\
15 & 2,55 & 186 \\
25 & 0,85 & 194 \\
25 & $-0,48$ & 206 \\
$\infty$ & $-2,18$ & -
\end{tabular}

Trockensubstanz: $37,5 \%$.
(Tab. 5.) IV.

70 Stunden vorbehandelt. Temp. $18,0^{\circ}$.

\begin{tabular}{c|r|c}
\hline \hline Minuten & \multicolumn{1}{|c|}{$a$} & $\mathrm{k} \cdot 10$ \\
\hline 0 & 6,75 & - \\
16 & 3,67 & 115 \\
25 & 2,45 & 114 \\
35 & 1,27 & 118 \\
$\infty$ & $-2,16$ & -
\end{tabular}

Trockensubstanz: $28,9 \%$.

(Tab. 7.) VI.

165 Stunden vorbehandelt. Temp. $19^{\circ}$.

\begin{tabular}{c|r|c}
\hline \hline Minuten & \multicolumn{1}{|c|}{$\alpha$} & $\mathrm{k} \cdot 10^{4}$ \\
\hline 0 & 6,85 & - \\
15 & 2,82 & 171 \\
25 & 0,97 & 183 \\
35 & $-0,36$ & 198 \\
$\infty$ & $-2,19$ & -
\end{tabular}

Trockensubstanz: $35,4 \%$.

(Tab. 9.) $\quad \mathrm{V}_{2}$.

$125+38$ Stunden vorbehandelt. Temp. $19^{\circ}$.

\begin{tabular}{c|r|c}
\hline Minuten & \multicolumn{1}{|c|}{$\alpha$} & $\mathrm{k} \cdot 10^{4}$ \\
\hline 0 & 6,85 & - \\
15 & 2,43 & 194 \\
25 & 0,55 & 207 \\
35 & $-0,62$ & 217 \\
$\infty$ & $-2,19$ & -
\end{tabular}

Trockensubstanz: $38,9 \%$. 
Chemische Zusammensetzung und Bildung der Enzyme. VIII. 103

(Tab. 10.) IV

$74+42$ Stunden vorbehandelt.

Temp. $1^{\circ}$.

\begin{tabular}{c|r|c}
\hline \hline Minuten & \multicolumn{1}{|c|}{$a$} & $\mathrm{k} \cdot 10^{4}$ \\
\hline 0 & 6,82 & - \\
15 & 3,05 & 157 \\
25 & 1,32 & 164 \\
35 & 0,26 & 162 \\
$\infty$ & $-2,18$ & -
\end{tabular}

Trockensubstanz: $32,6 \%$.

(Tab. 12.) $\Pi_{1}$.

$24+18$ Stunden vorbehandelt. Temp. $19^{\circ}$.

\begin{tabular}{c|r|c}
\hline \hline Minuten & $\alpha$ & $\mathrm{k} \cdot 10^{4}$ \\
\hline 0 & 6,75 & - \\
15 & 3,29 & 142 \\
26 & 1,50 & 149 \\
35 & 0,48 & 151 \\
$\infty$ & $-2,16$ & -
\end{tabular}

Trockensubstanz: $30,6 \%$.

(Tab. 14.) $\quad \Pi_{1} a_{1}$.

$24+18+18+43$ Stunden vorbehandelt.

Temp. 192 $^{\circ}$.

\begin{tabular}{r|r|c}
\hline \hline Minuten & \multicolumn{1}{|c|}{$\alpha$} & $\mathrm{k} \cdot 10^{4}$ \\
\hline 0 & 6,82 & - \\
15 & 2,85 & 168 \\
25 & 0,95 & 183 \\
35 & $-0,23$ & 190 \\
$\infty$ & $-2,18$ & -
\end{tabular}

Trockensubstanz: $35,5 \%$.
(Tab. 11.) IV

$74+115$ Stunden vorbehandelt. Temp. $19^{\circ}$.

\begin{tabular}{c|r|c}
\hline Minuten & \multicolumn{1}{|c|}{$\alpha$} & $\mathrm{k} \cdot 10^{4}$ \\
\hline 0 & 6,85 & - \\
16 & 3,12 & 144 \\
25 & 1,69 & 147 \\
35 & 0,40 & 155 \\
$\infty$ & $-2,19$ & -
\end{tabular}

Trockensubstanz: $30,0 \%$.

(Tab. 13.) $\quad \mathrm{Il}_{1} \mathrm{a}$.

$24+18+18$ Stunden vorbeh. Temp. $19^{\circ}$.

\begin{tabular}{c|r|c}
\hline Minuten & \multicolumn{1}{|c|}{$\alpha$} & $\mathrm{k} \cdot 10^{4}$ \\
\hline 0 & 6,75 & - \\
15 & 2,68 & 177 \\
25 & 1,00 & 180 \\
35 & 0,12 & 169 \\
$\infty$ & $-2,16$ & -
\end{tabular}

Trockensubstanz: $34,0 \%$.

(Tab. 15.) $\quad \mathrm{II}_{1} \mathrm{a}_{12}$.

$24+18+18+43+18$ Stunden vorbehandelt.

Temp. $1^{\circ}$.

\begin{tabular}{c|r|c}
\hline Minuten & \multicolumn{1}{|c|}{$\alpha$} & $\mathrm{k} \cdot 10^{4}$ \\
\hline 0 & 6,82 & - \\
16 & 2,12 & 200 \\
25 & 0,55 & 207 \\
35 & $-0,60$ & 216 \\
$\infty$ & $-2,18$ & -
\end{tabular}

Trockensubstanz: $42,8 \%$. 
(Tab. 16.)

a)

\begin{tabular}{c|c|c|c|c|c}
\hline Ninuten & $\begin{array}{c}\text { Ohne } \\
\text { Vorbehand- } \\
\text { lung } \\
\text { ccm CO }\end{array}$ & $\begin{array}{c}\text { Vor- } \\
\text { behandelt } \\
\text { 46 Stunden } \\
\text { ccm CO, }\end{array}$ & $\begin{array}{c}\text { Vor- } \\
\text { behandelt } \\
\text { 70 Stunden } \\
\text { ccm CO, }\end{array}$ & $\begin{array}{c}\text { Vor- } \\
\text { behandelt } \\
\text { 118 Standen } \\
\text { ccm CO, }\end{array}$ & $\begin{array}{c}\text { Vor- } \\
\text { behandelt } \\
165 \text { Stunden } \\
\text { ccm CO, }\end{array}$ \\
\hline 60 & 35,5 & 33,5 & 24 & 5 & 1 \\
120 & 69 & 67 & 52 & 8 & 1,5 \\
180 & 108 & 101 & 74 & 12 & 1,8 \\
240 & 1.45 & 126 & 102 & 16 & 2 \\
300 & 177 & 152 & 125 & 25 & 2,1
\end{tabular}

(Tab. 17.)

B

B

C

C.

\begin{tabular}{r|c|c|c|c}
\hline \hline Minuten & $\begin{array}{c}\text { Vorbehandelt } \\
125+15 \text { Std. } \\
\text { ccm CO }\end{array}$ & $\begin{array}{c}\text { Vorbehandelt } \\
125+38 \text { Std. } \\
\text { ccm CO }\end{array}$ & $\begin{array}{c}\text { Vorbehandelt } \\
74+42 \text { Std. } \\
\text { ccm C0 }\end{array}$ & $\begin{array}{c}\text { Vorbehandelt } \\
74+115 \text { Std. } \\
\text { ccm CO }\end{array}$ \\
\hline 60 & 7 & 6 & 29 & 23 \\
120 & 12,5 & 11 & 59 & 45 \\
180 & 18 & 15,5 & 87 & 62 \\
240 & 25 & 19 & 107 & 83 \\
300 & 32 & 22,5 & 132 & 103
\end{tabular}

(Tab. 18.)

b)

c)

d)

e)

\begin{tabular}{r|c|c|c|c}
\hline \hline Minuten & $\begin{array}{c}\text { Vorbehandelt } \\
24+18 \text { Std. } \\
\text { ccm C0 }\end{array}$ & $\begin{array}{c}\text { Vorbehandelt } \\
24+18+18 \\
\text { Stunden } \\
\text { ccm C0 }\end{array}$ & $\begin{array}{c}\text { Vorbehandelt } \\
24+18+18 \\
+43 \text { Stunden } \\
\text { ccm C0 }\end{array}$ & $\begin{array}{c}\text { Vorbehandelt } \\
24+18+18 \\
+43+18 \text { St. } \\
\text { ccm CO }\end{array}$ \\
\hline 60 & 38 & 32 & 19 & 10,5 \\
120 & 72 & 61 & 35,5 & 21 \\
180 & 103 & 90 & 54,5 & 34 \\
240 & 130 & 113 & 79 & 47 \\
360 & 154 & - & - & 59
\end{tabular}


Chemische Zusammensetzung und Bildung der Enzyme. VIII. 105

(Tab. 19.)

1

\begin{tabular}{|c|c|c|c|c|c|c|c|}
\hline $\begin{array}{l}\text { Mi- } \\
\text { nuten }\end{array}$ & $\begin{array}{c}\text { Ohne } \\
\text { Vor- } \\
\text { behand- } \\
\text { lung } \\
\text { ccm } \mathrm{CO}_{2}\end{array}$ & $\begin{array}{c}\text { Vor- } \\
\text { behandelt } \\
21 \text { Std. } \\
\text { ccm } \mathrm{CO}_{2}\end{array}$ & $\begin{array}{c}\text { Vor- } \\
\text { behandelt } \\
69 \text { Std. } \\
\text { ccm } \mathrm{CO}_{2}\end{array}$ & 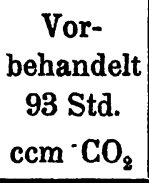 & $\begin{array}{c}\text { Vor- } \\
\text { behandelt } \\
117 \text { Std. } \\
\text { ccm CO }\end{array}$ & $\begin{array}{c}\text { Vor- } \\
\text { behandelt } \\
141 \text { Std. } \\
\text { ccm } \mathrm{CO}_{2}\end{array}$ & $\begin{array}{c}\text { Vor: } \\
\text { behandelt } \\
165 \mathrm{Std} . \\
\mathrm{ccm} \mathrm{CO}\end{array}$ \\
\hline 60 & 36,5 & 43 & 30 & 22 & 15 & 11 & 2 \\
\hline 120 & 72 & 85 & 59 & 44 & 31,5 & 20,5 & 3,5 \\
\hline 180 & 107 & 122 & 88 & 67 & 52 & 33 & 5,5 \\
\hline 240 & 137 & 155 & 117 & 89 & 70,5 & 46 & 7,5 \\
\hline 300 & 167 & 181 & 141 & 112 & 87 & 60 & 9,5 \\
\hline
\end{tabular}

(Tab. 20.)

2

\begin{tabular}{c|c|c|c|c}
\hline \hline Minuten & $\begin{array}{c}\text { Ohne } \\
\text { Vorbehand- } \\
\text { lung } \\
\text { ccm CO }\end{array}$ & $\begin{array}{c}\text { Vorbehandelt } \\
5 \text { Stunden } \\
\mathrm{ccm} \mathrm{CO}_{2}\end{array}$ & $\begin{array}{c}\text { Vorbehandelt } \\
22 \text { Stunden } \\
\text { ccm CO }\end{array}$ & $\begin{array}{c}\text { Vorbehandelt } \\
46 \text { Stunden } \\
\mathrm{ccm} \mathrm{CO}_{2}\end{array}$ \\
\hline 60 & 36 & 48 & 41,5 & 35 \\
120 & 76 & 92 & 83 & 67 \\
180 & 114 & 139 & 121 & 97 \\
240 & 148 & - & 155 & 123 \\
300 & 187 & - & 182 & 147
\end{tabular}

(Tab. 21.)

3

\begin{tabular}{|c|c|c|c|c|c|}
\hline Minuten & $\begin{array}{c}\text { Ohne } \\
\text { Vorbehand- } \\
\text { lung } \\
\text { ccm CO} \\
\end{array}$ & $\begin{array}{c}\text { Vor- } \\
\text { behandelt } \\
23 \text { Stunden } \\
\text { ccm } \mathrm{CO}_{2}\end{array}$ & $\mid \begin{array}{c}\text { Vor- } \\
\text { behandelt } \\
45 \text { Stunden } \\
\text { ccm } \mathrm{CO}_{2}\end{array}$ & $\begin{array}{c}\text { Vor- } \\
\text { behandelt } \\
69 \text { Stunden } \\
\mathrm{ccm} \mathrm{CO}_{2}\end{array}$ & $\begin{array}{c}\text { Vor- } \\
\text { behandelt } \\
93 \text { Stunden } \\
\mathrm{ccm} \mathrm{\textrm {CO } _ { 2 }} \\
\end{array}$ \\
\hline 60 & 38 & 46 & 32 & 15 & 4 \\
\hline 120 & 78 & 89 & 65 & 30,5 & 8 \\
\hline 180 & 118 & 124 & 94 & 46,5 & 12 \\
\hline 240 & 1 ว̄อ & 161 & 125 & 65,5 & 19 \\
\hline 300 & 191 & 189 & 153 & 88,5 & 30 \\
\hline
\end{tabular}

Die Mittelwerte der in den Tabellen 1-15 angegebenen Inversionskonstanten findet man in der Fig. 1 zusammengestellt. $\mathrm{Zu}$ den gleichzeitig angestellten Gärungsversuchen entnahmen 

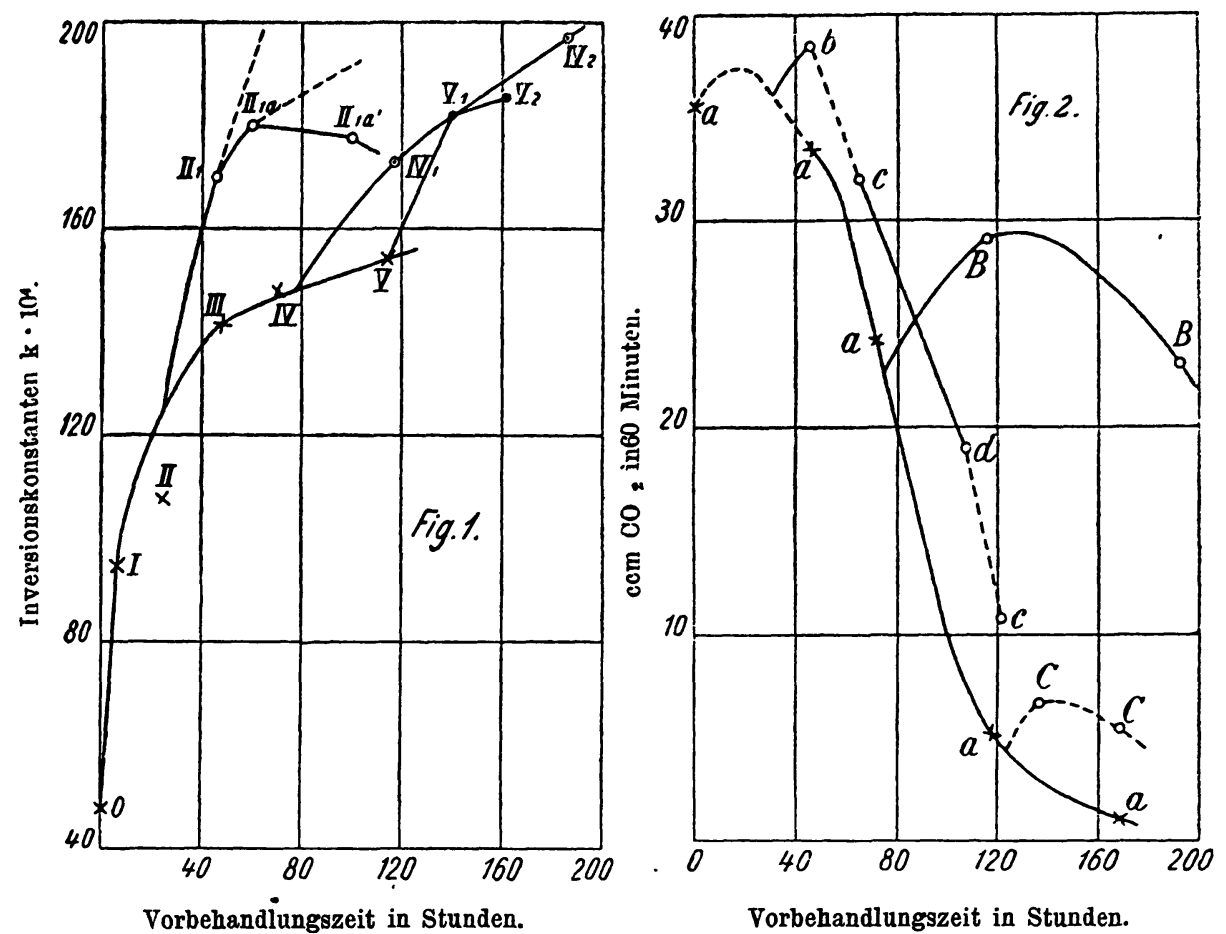

wir die Hefemengen denselben Kulturkolben, welche auch die Hefe für die Inversionsversuche lieferten, so daß die beiden Versuchsserien sich auf genau die gleiche Hefe beziehen. Diese Gärversuche sind in den Tabellen 16-18 wiedergegeben und in der Fig. 2 zusammengefaßt. Den einzelnen Versuchen a-e sowie $B$ und $C$ entsprechen in dieser Figur die so bezeichneten Punkte. Der -Vergleich der Fig. 1 und 2 gibt also ein vollständiges Bild von der gleichzeitigen Veränderung der Inversionsund Gärkraft bei gleicher Vorbehandlung der Hefe.

Wie unmittelbar ersichtlich, besteht durchaus keine Parallelität zwischen der gleichzeitigen Änderung der beiden Enzymwirkungen durch die Vorbehandlung. Während z. B. das Inversionsvermögen der Hefe nach 40stündiger Vorbehandlung auf mehr als das 3 fache steigt, ist in der gleichen Zeit die Gärkraft geringer als zu Anfang der Vorbehandlung geworden. Während dann weiter die Inversionskraft in den folgenden 
Tagen immer noch erheblich zunimmt, wird gleichzeitig die Gärkraft ganz außerordentlich stark geschwächt, so daß, wenn wir das Verhältnis der beiden Enzymwirkungen in den in den Figuren gewählten relativen Einheiten ausdrücken, der Zahlenwert dieses Verhältnisses beträgt:

Vor Beginn der Vorbehandlung Inversionsk. : Gärkraft

Nach 100 stündiger Vorbehandlung $150: 10=15,0$.

Es ist nun zunächst zu untersuchen, worauf die starke Erniedrigung der Gärkraft bei unserer Vorbehandlung beruht, und $\mathrm{ob}$ eine eventuelle Vermehrung der Zymase durch das Auftreten eines Hemmungskörpers verdeckt wird.

Daß sich die Gärwirkung der Hefe nicht in dem Maße erhöhen läßt, wie dies nach den Ergebnissen des hiesigen Laboratoriums für die Inversionswirkung der Fall ist, war schon nach einer Reihe älterer Versuche zu erwarten. Insbesondere sind hier die Untersuchungen von $\mathrm{H}$. Lang ${ }^{1}$ ) aus dem Institut für Gärungsgewerbe zu erwähnen und eine Arbeit von Buchner und Klatte. ${ }^{2}$ ) Aus den Versuchen der genannten Forscher geht in guter Übereinstimmung hervor, daß Vermehrung der Gärkraft durch das sogenannte Regenerationsverfahren im Umfang von 30-100\% auftritt, in höherem Maße aber nur dann, wenn die Gärkraft bezw. der Zymasevorrat im Ausgangsmaterial sehr gering war.

Wir machen hier noch besonders auf die Form der Gärkraftkurven der Fig. 2 und 3 aufmerksam. Es tritt bei der Vorbehandlung eine anfängliche Steigerung ein, welche sich auf die ersten 10-20 Stunden

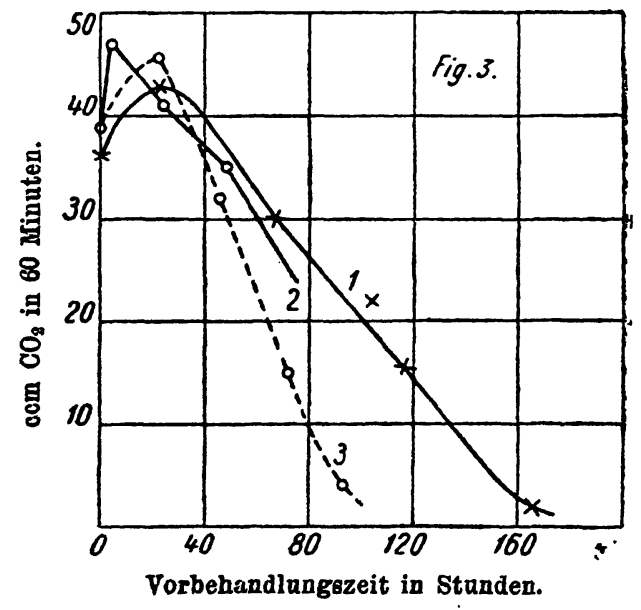

1) Wochenschrift für Brauerei, Bd. 24, S. 417, 1907.

2) Biochem. Zeitschrift, Bd. 9, S. 415, 1908. 
erstreckt, und sich bei jeder Überimpfung auf eine neue Menge der Nährlösung, also in einen neuen Kolben wiederholt. (Man beobachte in Fig. 2 die Punkte b, B und C.) Diese anfängliche Steigerung der Gärkraft, welche etwa in den Zeitraum der nach der Einführung der Hefe eintretenden Gärung fällt, wurde noch durch besondere Versuche näher studiert, welche in den Tabellen 19-21 enthalten und in der Fig. 3 zusammengefaßt sind. Auch bei diesen Versuchen tritt deutlich das Maximum der Gärwirkung innerhalb der ersten 24 Stunden zutage.

Die Versuchsbedingungen der drei Versuche 1, 2 und 3 dieser Figur unterscheiden sich, wie Seite 100 erwähnt, hinsichtlich der Zusammensetzung der Nährlösung, indem bei den Versuchen 1 und 2 die Nährlösung bedeutend weniger $\mathrm{NH}_{4}$ enthielt als bei Versuch 1. Diese Versuche mögen nur als für weitere Arbeiten orientierende betrachtet werden. Trotz den sehr wertvollen Versuchen von Lange lassen sich bis jetzt sehr wenig Schlüsse über die Erhöhung der Gärwirkung durch die Vorbehandlung ziehen.

\section{Zusammenfassung.}

Als Zusammenfassung unserer Versuche können die Kurven der Figuren 1-3 angesehen werden. Sie besagen, daß durch die von uns angewandte Vorbehandlung eine Vermehrung des enzymatischen Inversionsvermögens der Hefe eintritt,

welche weder als eine Anpassung aufgefaßt werden kann, da das Verweilen der Hefe in der Lösung des Spaltproduktes keine geringere Wirkung hervorruft als die Vorbehandlung mit dem Substrat Rohrzucker,

welche anderseits auch nicht auf eine allgemeine Erhöhung der vitalen Tätigkeit zurückgeführt werden kann, da gleichzeitig die Gärkraft sehr stark abnimmt,

sondern als eine Erscheinung eigener, noch unbekannter Art anzusehen ist. 